\title{
UNIVERSITARIOS Y RESPONSABILIDAD SOCIAL
}

\author{
Gracia Navarro' ${ }^{1}$ Paula Boero ${ }^{2}$, Gladys Jiménez ${ }^{3}$, Liliana \\ Tapia $^{4}$, Reinier Hollander ${ }^{5}$, Arturo Escobar ${ }^{6}$, Margarita \\ Baeza $^{7}$ y Álvaro Espina
}

\section{RESUMEN}

Esta investigación tuvo como objetivo conocer la frecuencia e intencionalidad del comportamiento socialmente responsable en un grupo de 5.515 estudiantes de seis universidades chilenas. Se utilizó el cuestionario CACSR (Davidovich, Espina, Navarro, 2005), compuesto por 2 escalas que miden 10 categorías de comportamiento y 3 categorías de intención. Los resultados muestran baja autoatribución de comportamientos socialmente responsables; sólo la categoría "convivencia social", cumple los requisitos necesarios: frecuencia y beneficio para todos. Estos resultados reflejan un empobrecimiento en la construcción de la identidad, en detrimento de las relaciones interpersonales basadas en consideraciones éticas de justicia y cuidado y, en consecuencia un progresivo desinterés por el bienestar de todos y el consiguiente perjuicio social. Luego, es imprescindible implementar estrategias que contrarresten las debilidades detectadas y promover la formación de ciudadanos responsables por el bienestar social.

Palabras clave: responsabilidad social, conducta socialmente responsable, ética, estudiantes universitarios.

\section{UNIVERSITY STUDENTS AND SOCIAL ACCOUNTABILITY}

\section{ABSTRACT}

This research was aimed to study frequency and intention of socially accountable behaviour in 5515 students from six Chilean universities. It used the CACSR questionnaire (Davidovich, Espina, Navarro, 2005) composed by two scales measuring 10 categories of behaviour and 3 categories of intention. Results showed a low self-attribution of socially accountable behaviour because only one (Social Interaction) out of these ten categories fulfils the requirements: frequency and benefit for everybody. These results reflect an impoverishment on the identity formation and a devaluation of interpersonal relationships based on ethical considerations of justice and care. Consequently, there is a progressive disinterest in the concept of welfare for everybody and an increasing possibility of social damage. It is critical to implement strategies to counteract the identified weaknesses and promote the formation of responsible citizens for social welfare.

Key words: social accountability, socially accountable behavior, ethics, university students.

1 Universidad de Concepción, Chile. Contacto: gnavarro@udec.cl

2-7 Universidad de La Frontera.

3 Pontificia Universidad Católica de Valparaíso.

4 Universidad de Valparaíso.

5 Universidad del Biobío.

6 Universidad Austral de Chile. 


\section{UNIVERSITARIOS Y RESPONSABILIDAD SOCIAL}

\section{Responsabilidad social, universidad y universitarios}

La responsabilidad social (RS) es conceptualizada como "la orientación de las actividades individuales y colectivas en un sentido que permita a todos igualdad de oportunidades para desarrollar sus capacidades, eliminando y apoyando la eliminación de obstáculos estructurales de carácter económico y social, así como los culturales y políticos que afectan o impiden ese desarrollo" (Urzúa, 2001, pp. 2-3). Desde la perspectiva de la psicología individual, Berman (1997) señala que se trata del compromiso personal con los demás, e incluye tres dimensiones necesarias para comprometerse con otros, esto es (a) entender que cada persona pertenece a una red social más amplia cuya influencia es decisiva en la construcción de la propia identidad; (b) mantener relaciones interpersonales basadas en consideraciones éticas de justicia y de preocupación por los otros, y (c) actuar con integridad, consistentemente con los propios valores, en otras palabras es la inversión personal en el bienestar propio, de otros y del planeta. En un plano más concreto, Navarro (2006) señala que como la RS es una abstracción, un valor, es necesario explicitar que una persona sólo es socialmente responsable si además de conocer el concepto lo ejerce y concreta a través de una conducta moral, esto es, mediante la realización de actos virtuosos que apunten en dirección al bien, a la supervivencia y a la felicidad del hombre y de la especie humana. En ese sentido el comportamiento socialmente responsable puede ser concebido como "aquellas conductas, que pueden darse en diferentes ámbitos, que tienen a la base una intención orientada hacia el bienestar de todos y que se ejercen con una determinada frecuencia" (Navarro, 2006, p. 86). Es decir, junto al desarrollo moral es necesario que las personas adquieran determinadas habilidades sociales que les permitan ejercer conductas pro sociales y comportamientos orientados a considerar tanto las necesidades propias como las de los otros (Davidovich, Espina, Navarro et al., 2005). 
En el plano educativo, la RS ha sido promovida por la UNESCO a partir de la Declaración de la Conferencia Mundial de Educación Superior de 1998 y ratificada en 2009, declarando en esta última ocasión que la RS es un compromiso de todos los actores involucrados y especialmente de los gobiernos. De manera más específica, en el ámbito de la educación superior demanda, de las instituciones, la RS para mejorar la comprensión de materias que presentan múltiples aristas, considerando las categorías sociales, económicas, científicas y culturales, y las habilidades para responder a ellas. Asimismo, indica que la educación superior debería incrementar su mirada interdisciplinaria y promover el pensamiento crítico y una ciudadanía activa para contribuir al logro del desarrollo sustentable, la paz, el bienestar y los derechos humanos, incluyendo la equidad de género. También señala que la educación superior no sólo debería proveer competencias sólidas al mundo presente y futuro, sino que debe contribuir a la educación de ciudadanos éticos, comprometidos con la construcción de la paz, la defensa de los derechos humanos y los valores de la democracia.

De la misma forma, el Proyecto Tuning Latinoamérica (2007), considera la "responsabilidad social y el compromiso ciudadano" dentro de las 27 competencias genéricas definidas como prioritarias para la educación superior en nuestro continente. Ello no es casualidad, porque son estas instituciones quienes forman a las elites intelectuales que a mediano plazo dirigirán los destinos de los países. De ellas surgen los profesionales y académicos que se espera tengan liderazgo en la sociedad. En ellas se deberían formar las personas -hombres y mujeres- encargadas de crear las condiciones humanas para que la responsabilidad y talentos del resto de la sociedad se desarrollen y se expresen al máximo. Específicamente en el caso de la universidad, forma parte de su misión crear conocimiento y formar los científicos, humanistas y profesionales orientados a satisfacer las necesidades de desarrollo de un país (Proyecto Universidad Construye País, 2006). Los temas como la pobreza, la desintegración social, el desarrollo del capital social y el desarrollo sustentable deberían estar en el centro de sus preocupaciones. Sin embargo, es posible observar que priman tendencias orientadas a la competitividad, el utilitarismo y el éxito personal en detrimento de los valores como el respeto, la 
fraternidad, la honestidad, la solidaridad y la responsabilidad para con la sociedad (Equipo Coordinador Universidad Construye País, 2002), produciéndose múltiples ejemplos de irresponsabilidad como el plagio, la corrupción, el mal ejercicio profesional, entre otros (Gardner, 2007). Comportamientos que finalmente traen aparejados la pobreza y el malestar de la sociedad en su conjunto. Luego, frente a estos inquietantes hechos surge la pregunta: iestán las universidades chilenas formando profesionales que actúen de forma socialmente responsable?

En relación con esta interrogante seis universidades pertenecientes al Consejo de Rectores de Chile aceptaron el desafío de responderla. Estas instituciones son las universidades de Concepción, Del Biobío, Austral de Chile, De Valparaíso, De La Frontera y Pontificia Universidad Católica de Valparaíso, las que con el apoyo del Ministerio de Educación de Chile a través del área de Mejoramiento de la Calidad y Equidad de la Educación Superior (MECESUP) ejecutaron el Proyecto MECESUP UCO 0303 "Formación de profesionales con valores, actitudes y comportamientos necesarios para ejercer la responsabilidad social". En este contexto, las primeras preguntas para responder fueron ¿cuáles son las conductas de los universitarios chilenos respecto de la responsabilidad social? y ¿cuáles son las intenciones asociadas a este ejercicio? Para eso se llevó a cabo un diagnóstico con el objetivo de conocer los comportamientos relacionados con el ejercicio de la RS y la intencionalidad reportada, al ejercerlos, en un grupo de universitarios chilenos.

\section{Método}

\section{Participantes}

La muestra estuvo constituida por 5.515 estudiantes pertenecientes a 56 carreras de las seis universidades, que voluntariamente se adscribieron al Proyecto MECESUP UCO 0303. Esta cifra representa un $8,53 \%$ del total de alumnos matriculados en las seis instituciones de educación superior. Los sujetos tienen una edad promedio de 20,37 años y una media de 18 años. De ellos, un 56,2\% es de sexo femenino y un $43,2 \%$ de sexo masculino. Un $22 \%$ de los encuestados pertenecen 
a las carreras clasificadas dentro del área temática físico-matemática; un $35,9 \%$ son de carreras del área químico-biológica, y un $42 \%$ al área de ciencias sociales y humanidades. Finalmente, un $67,1 \%$ corresponde a estudiantes de primer año y un 32,9\% a cuarto año, muestreados con el fin de establecer comparaciones entre ambos grupos.

\section{Instrumento}

Se utilizó el cuestionario para evaluar comportamientos socialmente responsables en estudiantes universitarios (CACSR), que fuera elaborado en la Universidad de Concepción por Davidovich, Espina, Navarro et al. (2005). Está compuesto por dos escalas que miden diez categorías de comportamiento y tres categorías de intención. Las escalas son:

1) Frecuencia de comportamiento socialmente responsable (E1): consta de 40 ítems con respuestas tipo likert de cinco opciones, en donde el puntaje asignado, varía desde 1 a 5 puntos, con nunca= 1 punto; casi nunca $=2$ puntos; a veces $=3$ puntos; casi siempre $=4$ puntos; siempre $=5$ puntos. A mayor puntaje, mayor es el reporte de frecuencia de una determinada conducta. Anexo A.

2) Intencionalidad del comportamiento socialmente responsable (E2): considera los 40 ítems anteriores y pregunta por la intención al elegir esa opción. Las respuestas se relacionan con las categorías: beneficio personal = 1 punto; no tiene clara la intención $=2$ puntos; beneficio para otros $=3$ puntos; beneficio para todos $=$ 4 puntos. Anexo B.

Las categorías de comportamiento son:

"Responsabilidad académica": incluye los comportamientos vinculados al ejercicio de una vida universitaria acorde con las exigencias y/u oportunidades que demanda y/u ofrece cada carrera.

"Actividades de voluntariado": alude a los comportamientos relativos al ejercicio de una vida con un sentido solidario que atienda y satisfaga en forma directa las carencias y necesidades de los otros.

"Ayuda social": se refiere a los comportamientos vinculados a la solidaridad que indirectamente atienden y satisfacen las carencias y necesidades de los otros. 
"Actividades religiosas": incluye los comportamientos relativos al ejercicio de una vida espiritual basada en los valores de la propia religión, sin ir en desmedro de los valores y creencias religiosas de los demás.

"Convivencia social": alude a los comportamientos referidos al ejercicio de una vida en comunidad acorde con las normas y necesidades individuales y grupales.

"Responsabilidad cívico-universitaria": se refiere a los comportamientos vinculados al ejercicio de los derechos y deberes cívicos que cada estudiante posee como miembro de una comunidad universitaria y social en general.

"Autocuidado": incluye los comportamientos vinculados al resguardo de la salud física y psíquica.

"Desarrollo cultural": incluye los comportamientos relativos a la formación integral de las personas, pero que no se relacionan directamente con su área de formación académica.

"Ecología y medioambiente": se refiere a los comportamientos vinculados al cuidado del ambiente.

"Respeto por los espacios compartidos": alude a la utilización de espacios públicos de forma responsable.

La confiabilidad medida a través del Coeficiente de Alpha de Cronbach es de 0,82 para la E1, y de 0,61 para la E2. La validez fue verificada mediante el método interjueces.

\section{Procedimiento}

La aplicación se realizó en paralelo en las universidades integrantes del proyecto durante el primer semestre lectivo. El procedimiento fue estandarizado, a través de manuales de procedimiento tanto para los encuestadores, coordinador de aplicación, como el coordinador general de evaluación en cada una de las universidades participantes. La aplicación fue realizada en forma colectiva en sala de aula. Allí los estudiantes fueron informados acerca del objetivo de la evaluación, su carácter voluntario y la confidencialidad en el tratamiento de los datos. La aplicación del CACSR tuvo una duración aproximada de 40 minutos. 


\section{Resultados}

Los datos del conjunto de universidades, que fueron procesados de forma centralizada utilizando el software estadístico Statistical Analisys System (SAS), serán presentados primero de acuerdo con los porcentajes obtenidos según las alternativas de respuesta y las medias por categoría; luego, se expondrán los resultados según sexo, área temática y promoción de pertenencia académica. Las diferencias serán establecidas al interior de los grupos a través de pruebas t para grupos independientes, para sexo y promoción. Y un ANOVA de una vía para las diferencias entre las tres áreas temáticas.

Los resultados para la escala El (frecuencia de comportamiento), expuestos en la tabla n. ${ }^{\circ} 1$, muestran que los ítems que obtuvieron un mayor porcentaje de respuesta considerando las alternativas "casi siempre" y "siempre" fueron pago la universidad (aranceles, crédito universitario, devolver el crédito, matrícula y hacer uso honesto de las becas); cuido instalaciones públicas o universitarias como mobiliario, infraestructura, libros, etc., y asisto a clases.

Tabla n. ${ }^{\circ}$ 1. Ítem de la Escala 1 con mayor porcentaje de respuesta considerando la sumatoria de las alternativas "Siempre" y "Casi siempre".

\begin{tabular}{|l|c|c|}
\hline Ítem & $\mathrm{n}$ & $\%$ \\
\hline $\begin{array}{l}\text { 7. Pago la universidad (aranceles, crédito universitario, devolver el crédito, } \\
\text { matrícula y hacer uso honesto de las becas) }\end{array}$ & 3.927 & 71,2 \\
\hline $\begin{array}{l}\text { 35. Cuido instalaciones públicas o universitarias (mobiliario, infraestructura, } \\
\text { libros, etc.) }\end{array}$ & 3.927 & 71,2 \\
\hline 3. Asisto a clases & 3.861 & 70,0 \\
\hline
\end{tabular}

A la inversa, los ítems que obtuvieron un mayor porcentaje de respuesta considerando las alternativas "casi nunca" y "nunca", expuestos en la tabla n. ${ }^{\circ} 2$, fueron dono sangre; formo parte de alguna organización de ayuda social (Hogar de Cristo, Fundación Las Rosas, Regazo, etc.) y participo en campañas de cuidado del medioambiente (de recolección de basura, limpieza de lugares públicos, etc.). 
108 UNIVERSITARIOS Y RESPONSABILIDAD SOCIAL - Gracia Navarro, Paula Boero, Gladys Jiménez, Liliana Tapia, Reinier Hollander, Arturo Escobar, Margarita Baeza, y Álvaro Espina

Tabla n. ${ }^{\circ}$ 2. Ítem de la Escala 1 con mayor porcentaje de respuesta considerando la sumatoria de las alternativas "Casi nunca" y "Nunca".

\begin{tabular}{|l|c|c|}
\hline Ítem & $\mathrm{n}$ & $\%$ \\
\hline 2. Dono sangre & 3.468 & 62,9 \\
\hline $\begin{array}{l}\text { 30. Formo parte de alguna organización de ayuda social (Hogar de Cristo, } \\
\text { Fundación Las Rosas, Regazo, etc.) }\end{array}$ & 3.430 & 62,2 \\
\hline $\begin{array}{l}\text { 1. Participo en campañas de cuidado del medioambiente (de recolección de } \\
\text { basura, limpieza de lugares públicos, etc.) }\end{array}$ & 3.386 & 61,4 \\
\hline
\end{tabular}

Por categoría, en orden decreciente, se observa que los estudiantes ejercitan "casi siempre" conductas socialmente responsables en las categorías relativas al "respeto por los espacios compartidos" (cuido espacios públicos), "responsabilidad académica" (estudio las asignaturas de mi carrera), "autocuidado" (evito contagiarme de enfermedades de transmisión sexual) y "convivencia social" (acepto a los demás independientemente de su sexo, raza, condición física, orientación sexual, etc.). Sólo "a veces" ejercen conductas relacionadas con las categorías: "desarrollo cultural" (participo en actividades culturales como conciertos, obras de teatro), "ecología y medioambiente" (boto la basura en los basureros de la calle, de los parques o de lugares públicos), "responsabilidad cívicouniversitaria" (participo de movimientos estudiantiles tales como federación de estudiantes, centros de alumnos) y "ayuda social" (dono dinero a campañas de ayuda social). Y "casi nunca" comportamientos asociados a las "actividades religiosas" (transmito valores religiosos propios a otras personas) y "actividades de voluntariado" (participo en actividades de voluntariado como construir mediaguas, visitar hogar de niños o de ancianos), como se observa en la tabla n. ${ }^{\circ} 3$.

Tabla n. ${ }^{\circ}$ 3. Media y desviación estándar de la escala El en estudiantes universitarios.

\begin{tabular}{|l|c|c|c|c|}
\hline Categorías & mín. & máx. & media & d.e. \\
\hline Respeto por espacios compartidos & 1,00 & 5,00 & 4,4978 & 0,50 \\
\hline Responsabilidad académica & 1,25 & 5,00 & 4,3070 & 0,50 \\
\hline Autocuidado & 1,25 & 5,00 & 4,2851 & 0,51 \\
\hline Convivencia social & 1,00 & 5,00 & 4,1896 & 0,52 \\
\hline Desarrollo cultural & 1,00 & 5,00 & 3,4514 & 0,69 \\
\hline Ecología y medioambiente & 1,25 & 5,00 & 3,3486 & 0,57 \\
\hline Responsabilidad cívico-universitaria & 1,00 & 5,00 & 3,2284 & 0,66 \\
\hline Ayuda social & 1,00 & 5,00 & 3,1221 & 0,59 \\
\hline Actividades religiosas & 1,00 & 5,00 & 2,2535 & 1,16 \\
\hline Actividades de voluntariado & 1,00 & 5,00 & 2,2333 & 0,79 \\
\hline Frecuencia comportamientos E1 & 1,93 & 4,82 & 3,4391 & 0,36 \\
\hline
\end{tabular}


En términos de la E2, como se observa en la tabla n. ${ }^{\circ} 4$, los ítems que obtuvieron un mayor porcentaje de respuesta considerando la intención predominante "beneficio personal" fueron evito contagiarme de enfermedades de transmisión sexual; asisto a clases; cumplo responsablemente tareas, trabajos y evaluaciones; participo en actividades culturales (ir a conciertos, ver obras de teatro, ir a exposiciones de obras de pintura, escultura, etc.); leo libros no relacionados con lo académico. En cambio, aquella conducta que ejecutan con la intención de beneficiar a "otros" es dono dinero a campañas de ayuda social, y por último, las conductas en donde la intención que predomina es el "beneficio para todos", los ítems que obtuvieron un mayor porcentaje de respuesta fueron: cuido espacios públicos (plazas, jardines, parques, monumentos, etc.); boto la basura en los basureros de la calle, de los parques o de lugares públicos y expreso mi opinión respecto a distintos temas, en diferentes contextos y con diferentes personas, como se observa en la tabla n. ${ }^{\circ} 5$.

Tabla n. ${ }^{\circ} 4$. Ítem de la Escala 2 según intención beneficio personal.

\begin{tabular}{|l|c|c|}
\hline Ítem & $\mathrm{n}$ & $\%$ \\
\hline 26. Evito contagiarme de enfermedades de transmisión sexual & 3.502 & 63,5 \\
\hline 3. Asisto a clases & 3.497 & 63,4 \\
\hline 12. Cumplo responsablemente tareas, trabajos y evaluaciones & 3.353 & 60,8 \\
\hline $\begin{array}{l}\text { 39. Participo en actividades culturales (ir a conciertos, ver obras de teatro, ir a } \\
\text { exposiciones obras de pintura, escultura, etc.) }\end{array}$ & 3.049 & 55,3 \\
\hline 27. Leo libros no relacionados con lo académico & 2.989 & 54,2 \\
\hline
\end{tabular}

Tabla n. ${ }^{\circ}$ 5. Ítem de la Escala 2 según intención beneficio para todos.

\begin{tabular}{|l|c|c|}
\hline Ítem & $\mathrm{n}$ & $\%$ \\
\hline 11. Cuido espacios públicos (plazas, jardines, parques, monumentos, etc.) & 3.171 & 57,5 \\
\hline $\begin{array}{l}\text { 10. Boto la basura en los basureros de la calle, de los parques o de lugares } \\
\text { públicos }\end{array}$ & 3.144 & 57,0 \\
\hline $\begin{array}{l}\text { 37. Expreso mi opinión respecto a distintos temas, en diferentes contextos y } \\
\text { con diferentes personas }\end{array}$ & 2.951 & 53,5 \\
\hline
\end{tabular}

Por categorías, se observa que predomina el "beneficio personal" en "autocuidado", "desarrollo cultural", "responsabilidad cívico-universitaria" y "responsabilidad académica". Luego, en "beneficio hacia otros" prevalecen las categorías "actividades religiosas", "ayuda social", "actividades de voluntariado" y "respeto por los espacios compartidos". Y por último, las categorías en que 
domina el "beneficio para todos" son "convivencia social" y "ecología y medioambiente".

Los resultados según sexo evidencian que las mujeres manifiestan una frecuencia de comportamiento socialmente responsable en promedio mayor $(\mathrm{M}=3,51, \mathrm{DE}=0,36)$ que la de los hombres $(M=3,35, D E=0,35)$, y en general, actúan con intenciones más colectivistas que estos últimos. La comparación de la prueba t para grupos independientes revela que estas diferencias son estadísticamente muy significativas en ambas escalas $(t=-16,165$, $\mathrm{p}=0,000)$ y $(\mathrm{t}=-9,671, \mathrm{p}=0,000)$. Los análisis además muestran que las mujeres obtienen una frecuencia mayor de comportamiento socialmente responsable en todas las categorías excepto en "desarrollo cultural", en donde no se diferencian de los hombres. Asimismo, los resultados revelan que las mujeres actúan de forma más colectivistas que los hombres en las categorías "actividades de voluntariado" $(\mathrm{t}=-6,767 ; \mathrm{p}=0,000)$, "ayuda social" $(\mathrm{t}=-12,530 ; \mathrm{p}=0,000)$, "actividades religiosas" ( $\mathrm{t}=-8,759 ; \mathrm{p}=0,000)$, "convivencia social" $(\mathrm{t}=-4,249 ; \mathrm{p}=0,000)$, "ecología y medio ambiente" $(\mathrm{t}=-5,803$; $\mathrm{p}=0,000)$ y "respeto por los espacios compartidos" ( $t=-6,474$; $p=0,000)$. No hubo diferencias, entre mujeres y hombres, en las intenciones reportadas para las categorías "auto cuidado" ( $\mathrm{t}=$ -1,526; $\mathrm{p}=0,127)$, "desarrollo cultural" $(\mathrm{t}=-0,036 ; \mathrm{p}=0,971)$ y "responsabilidad académica" ( $\mathrm{t}=0,462 ; \mathrm{p}=0,644)$.

En relación con las áreas de conocimiento se observa que los estudiantes del área ciencias sociales y humanidades son quienes obtienen una mayor frecuencia de comportamiento socialmente responsable $(\mathrm{M}=3,48, \mathrm{DE}=0,37)$, seguidos por los del área químicobiológica ( $M=3,46, D E=0,35)$, y luego los estudiantes del área físicomatemática ( $\mathrm{M}=3,33, \mathrm{DE}=0,35)$. Y comparadas las medias mediante un ANOVA, se encontró que estos últimos, muestran diferencias estadísticamente muy significativas respecto a los otros dos grupos $(\mathrm{F}=71,466 ; \mathrm{p}=0,000)$, además de intenciones más individualistas $(\mathrm{F}=14,564 ; \mathrm{p}=0,000)$.

Los resultados según promoción indican que los estudiantes de cuarto año obtienen una media mayor en comportamiento 
socialmente responsable y una intencionalidad más colectivista que los estudiantes de primer año, cuando se los compara a través de una prueba $t$ para muestras independientes $(t=-2,329 ; p=0,021)$ $y(t=-3,327 ; p=0,001)$.

\section{Discusión}

Considerando que el comportamiento socialmente responsable es aquel que tiene a la base una intención orientada hacia el bienestar de todos y que se ejerce con una determinada frecuencia (Navarro, 2006), el objetivo de este diagnóstico fue precisamente conocer cuáles eran esos comportamientos y la intencionalidad subyacente a su ejercicio en un grupo de universitarios chilenos. Se observa que los encuestados reportan cumplir con ambos requisitos en todos los ítem de una sola de las diez categorías que mide el cuestionario, esta es "convivencia social". Además, se cumplen de forma parcial estas condiciones en cinco categorías: "ecología y medioambiente", "respeto por los espacios compartidos", "ayuda social", "actividades de voluntariado" y "actividades religiosas" y se excluyen cuatro: "autocuidado", "desarrollo cultural", "responsabilidad académica" y "responsabilidad cívico-universitaria", que aunque los estudiantes reportan ejecutar con alguna regularidad, están orientadas hacia "beneficio personal", luego no es posible considerarlas como tales.

Observamos así que los estudiantes universitarios chilenos encuestados se atribuyen conductas socialmente responsables en los comportamientos referidos al ejercicio de una vida en comunidad acorde con las normas y necesidades individuales y grupales, lo que es muy alentador, porque está dimensión habla de la tolerancia de los jóvenes a la diversidad, a su respeto hacia otros en la comunicación, respeto de las normas sociales y, al mismo tiempo, de la percepción de que son capaces de expresar sus opiniones sobre temas diversos con libertad: es decir; junto con el respeto a otros existe la apreciación de que pueden ser respetados.

Sin embargo, en las otras categorías, el autorreporte de comportamiento socialmente responsable es parcial y varía desde un cumplimiento casi total de ambos requisitos en los ítems 
que conforman la dimensión -como en la categoría "ecología y medioambiente"- hasta ningún ítem, como ya se ha señalado. Los ítems que cumplen estas condiciones para la categoría "ecología y medioambiente" revelan que los estudiantes están preocupados tanto por cuidar el medioambiente (participo en campañas de cuidado del medioambiente de recolección de basura, limpieza de lugares públicos, etc.) como por evitar su daño (evito usar productos que contaminen el ambiente, como aerosoles, envases no reciclables, tubos de escape en mal estado, etc.). Sólo se excluye "utilizo racionalmente recursos naturales (agua, luz, gas, cuidar espacios naturales, etc.)", que aunque se ejecuta "casi siempre", su intencionalidad curiosamente es vista principalmente en función del "beneficio hacia otros", pese a que de manera secundaria se vislumbre también el "beneficio para todos". Este resultado pudiera ser atribuido a que la publicidad en Chile enfoca los beneficios del cuidado de los recursos naturales en el futuro, es decir, para que las próximas generaciones (otros) puedan disfrutar de lo que tenemos hoy, pero en ningún caso a que sea bueno para "todos" desde ahora. Otra explicación pudiera estar dada en función de que los promotores de las campañas de cuidado del medioambiente a menudo son empresas, y se genera la percepción de que hay más intereses involucrados que los exclusivamente asociados a la responsabilidad social.

En las demás categorías sólo se cumplen los requisitos de autoatribución de comportamiento socialmente responsable en un ítem por categoría, lo que revela, en general, un bajo grado de este constructo. Este hecho es preocupante, pues si consideramos que la autoatribución de comportamientos socialmente responsables implicaría una predisposición a realizarlos, ésta sería más bien baja. Así, cuando se revisa la media de comportamiento socialmente responsable para la muestra completa $(\mathrm{M}=3,43, \mathrm{DE}=0,36)$, se ve que los estudiantes reportan comportarse de forma socialmente responsable "sólo a veces", y que la intencionalidad subyacente está centrada entre el "beneficio hacia otros" y el "beneficio personal" (M $=2,48, \mathrm{DE}=1,27$ ), hecho que nos muestra que no se está pensando en la sociedad en su conjunto, sino que se piensa en función de "yo" y de "los otros" significativos como polos importantes, y muy poco en función de "todos". 
Una explicación, puede encontrarse en que los universitarios chilenos, al igual que los jóvenes vascos del estudio de Cortés (2002), se encuentren mayoritariamente en un estadio moral de conformidad de las expectativas y las relaciones interpersonales, en que la comprensión de los valores se hace en función del grupo colectivo. Y a pesar de que tienen en cuenta las expectativas de los demás y su responsabilidad con la sociedad, su razonamiento siempre está en función de la aprobación social de los otros y no en razón de principios universales. Asimismo, en el citado estudio se observó que los conflictos sociomorales se sitúan, casi completamente, en los entornos proximales, es decir, en los ámbitos de amistad y familia, y por tanto, se reducen las posibilidades de reflexionar y actuar pensando en el bienestar social, dando origen a lo que Tironi (2005) ha denominado la "privatización de las relaciones sociales", esto es, una tendencia de las personas a percibir que las características de su vida son personales, situación que lleva a los individuos a afirmar que lo único que importa es la suerte individual y la del entorno familiar. Pues, como observamos a través de los resultados, los comportamientos en los cuales se pudiera hipotetizar que subyacería una intención de beneficio colectivo que se orienta hacia "todos", como en las categorías de "ayuda social" y "actividades de voluntariado", sólo se orientan hacia "los otros", probablemente los otros conocidos; porque, como reveló un reciente estudio con jóvenes brasileños, ellos reportan tener más confianza en personas de su círculo privado (padres y amigos) y sienten que son éstas una fuente de influencia mayor que la escuela, los medios de comunicación y la religión. En cambio, el espacio público les parece amenazador, pues en él perciben más adversarios que amigos, más agresividad que diálogo (La Taille, 2006).

Estos datos también pueden servir de referentes para comprender las diferencias en los resultados obtenidos al comparar estudiantes de primer y cuarto año, ya que como se recordará los últimos obtienen una media más alta en la frecuencia de comportamiento socialmente responsable y una intencionalidad más colectivista que los primeros. Y esto es totalmente coherente, pues el círculo de relaciones y acción de los primeros es más reducido que el de los segundos: en general está limitado a la familia y a los amigos, quienes casi siempre son los compañeros del establecimiento educacional de pertenencia, por 
tanto, de ambientes más bien homogéneos, y luego, la posibilidad de apreciar otras realidades, otras necesidades, valores y opciones de vida son muy limitadas, y recién con el ingreso a la universidad pueden llegar a ampliarse con la eventualidad de despertar un sentido de ser social y de respuesta hacia una comunidad más amplia de la cual se forma parte.

Otra explicación, que no excluye a las anteriores, es que efectivamente, como señalan Davidovich, Espina, Navarro et al. (2005), los estudiantes tengan pocas oportunidades de participar en actividades que contribuyan al "bienestar de todos", y de allí la dificultad para integrar el sentido de que sus acciones $u$ omisiones involucran y afectan a la sociedad en su conjunto. Pues, como sugieren algunos datos actuales, el voluntariado y otros tipos de conductas pro sociales son importantes para la reflexión sobre temas como la justicia y el bienestar, contribuyendo al sentido de identidad de las personas (Pasupathi y Wainryb, 2010). Y aunque hay que hacer notar que esta medición fue realizada antes del terremoto de febrero de 2010, luego del cual hubo un gran movimiento que involucró a miles de universitarios en trabajos voluntarios de reconstrucción y apoyo a los damnificados, hecho que podría haber modificado los datos presentados, se refiere a un fenómeno único de una magnitud excepcional que no constituye una regularidad en términos de las actividades que normalmente desarrollan los universitarios. Sin embargo, bien valdría la pena hacer una medición actual para saber cuánto influyó ese fenómeno en las acciones socialmente responsables de los estudiantes, así como saber cuáles han sido otras repercusiones y cuál será la latencia de este fenómeno. Es de suma importancia, pues, incorporar cuando sea necesario, y fortalecer cuando ya exista, este aspecto dentro de la formación universitaria, de manera que haya una estrecha relación con la actividad profesional y esta unión sea duradera y persista durante la vida profesional futura tanto en términos de acción como de reflexión.

Asimismo, las diferencias observadas entre las áreas temáticas de conocimiento (ciencias sociales y humanidades, químico-biológica y físico-matemática) se pueden atribuir por un lado al mayor contacto con las personas que las dos primeras áreas requieren, en términos de 
formación profesional; es decir, carreras como psicología, educación parvularia, enfermería y medicina veterinaria, entre otras, tienen la oportunidad de interactuar con otros significativos desde muy temprano en su carrera, lo que ayuda a desarrollar conductas pro sociales y cooperativas como hemos visto. En detrimento de las carreras del área físico-matemática, que sólo hasta una etapa muy avanzada de su currículum tienen esta oportunidad. A eso se suma la mayor cantidad de varones que cursa estas carreras, pues como se vio en los resultados la variable sexo reporta diferencias significativas entre hombres y mujeres: esto es, una mayor frecuencia de comportamiento socialmente responsable e intenciones más orientadas hacia el bienestar de otros y de todos, en las mujeres. Estas diferencias pueden ser explicadas a partir de las investigaciones realizadas por Chodorow (1974) y Gilligan $(1992,1994)$, en las cuales se propone que estos contrastes se derivan de un desarrollo psicológico femenino diferente, que lleva a una construcción moral distinta que la masculina y que sería la razón de por qué las mujeres actúan de una forma que revela primordialmente una ética de cuidado por sobre una ética de justicia, más característica de los varones. De esta forma, las mujeres se definirían a sí mismas en relación con el mundo describiéndose por medio de acciones que las ponen en contacto con los demás, elaborando nexos por medio de su capacidad de dar ayuda, es decir: su yo es delimitado por la conexión con otros. De manera que en sus acciones morales prevalece la premisa central de "no violencia": nadie debe ser perjudicado, por el contrario, debe ser cuidado, incluido y respetado (Gilligan, 1994).

Por otra parte, fue interesante observar que no existían estas diferencias en la frecuencia de comportamiento socialmente responsable de la categoría "desarrollo cultural", que alude a los comportamientos relativos a la formación integral de las personas que no se relacionan directamente con su área de formación académica. Y más interesante aun fue constatar que en ambos sexos este aspecto tiene una orientación de beneficio personal, es decir, es vivenciado de forma en que la ganancia es sólo individual y no incumbe ni siquiera al entorno más próximo, ni menos a la sociedad en general. En resumen, este aspecto es vivenciado de forma totalmente privada. 
También prevaleció el beneficio personal en las categorías de "autocuidado", "responsabilidad cívico-universitaria" y "responsabilidad académica", lo que pudiera explicarse en función de que corresponden a dimensiones que por un lado son de un ámbito muy próximo a sí mismos, esto es, el propio cuerpo, la universidad y el propio ejercicio ciudadano, y por otro, el énfasis que la misma sociedad ha puesto sobre la preocupación individual que las personas deben tener respecto de estos aspectos y que señala Cortina (2010), inspirada en Mcpherson (1979), responde a la modernidad y con ella a la emergencia de la "razón subjetiva", en donde, el sujeto es concebido como propietario de su persona y de sus capacidades, sin deber nada a la sociedad. Bajo este concepto, dice Cortina, el individuo será libre en la medida que es propietario de sí mismo, de sus capacidades y del producto de sus capacidades, sin depender de la voluntad de los demás. Así, los hombres se consideran libres e iguales, y las relaciones que puedan surgir con otros estarán siempre evaluadas en función del propio beneficio, como también se puede constatar a través de esta investigación. Un reflejo de esta premisa es, como señalan Davidovich, Espina, Navarro et al. (2005), la fuerte competitividad académica inculcada desde la enseñanza primaria, en la que prevalece una perspectiva individual, en la que "ser mejor", ser "exitoso" y estar por encima de los otros se superpone a los intereses colectivos de bienestar de una sociedad, de un país o de la humanidad, es decir, sólo se trabaja para sí.

Finalmente, se puede concluir que la autoatribución de comportamientos socialmente responsables en los estudiantes universitarios chilenos encuestados es baja y que sólo se evidencia con claridad en la dimensión "convivencia social", una de las diez categorías medidas. Esta realidad plantea como desafíos, por un lado, conocer y comprender mejor este concepto a través del examen de los valores que subyacen en él y las actitudes que modelan este tipo de comportamiento; y por otro lado, se hace imprescindible implementar una serie de estrategias que contrarresten las debilidades detectadas, como la tendencia creciente al individualismo y la consiguiente percepción estrecha del entorno y que nos impide ser ciudadanos del mundo; a decir de Berman (1997): entender que cada persona pertenece a una red social más amplia cuya influencia es decisiva 
en la construcción de la propia identidad; mantener relaciones interpersonales basadas en consideraciones éticas de justicia y de preocupación por los otros y actuar con integridad, consistentemente con los propios valores, es decir, la inversión personal en el bienestar propio, de otros y del planeta. En otras palabras, con Responsabilidad Social.

\section{Referencias bibliográficas}

Berman, S. (1997) Children's social consciousness and the development of social responsibility. New York: New York State University Press.

Chodorow, N. (1974) Family structure and feminine personality. In M.Z. Rosaldo and L. Lamphere, eds., Woman, culture and society. Stanford: Stanford University Press.

Cortés, A. (2002) Contribución de la psicología ecológica al desarrollo moral. Un estudio con adolescentes. [Versión electrónica] Anales de Psicología, (18), pp.11-134.

Cortina, A. (2010) Ética sem moral. São Paulo: Martins Martins Fontes.

Davidovich, M. P.; Espina, Álvaro; Navarro, Gracia, et al. (2005) Construcción y estudio piloto de un cuestionario para evaluar comportamientos socialmente responsables en estudiantes universitarios. Revista de Psicología de la Universidad de Chile, (14) 1, pp.125-139.

Equipo Coordinador Universidad Construye País (2002) Marco Conceptual sobre la Responsabilidad Social Universitaria; Santiago, Chile: Autor.

Gilligan, C. (1992) Uma voz diferente: Psicologia da diferença entre homens e mulheres da infância a idade adulta. Rio de Janeiro: Rosa dos Tempos.

Gilligan, C. (1994) La moral y la teoría. Psicología del desarrollo femenino; México DF: Fondo de Cultura Económica.

La Taille, Y. (2006) Ética e Moral. Porto Alegre: Artmed.

Macpherson, C. B. (1979) La teoría política del individualismo posesivo. Barcelona: Fontanella.

Navarro, G.; Jiménez, G.; Tapia, L., et al. (2003) Formación de profesionales con valores, actitudes y comportamiento que favorezcan el ejercicio de su responsabilidad social (Proyecto MECESUP UCO 0303).

Navarro, G. (2006) Comportamiento socialmente responsable. En: Responsabilidad social universitaria, una manera de ser universidad, 
UAH-CIDE (2006) Encuesta a Actores Educativos, disponible en: www.cide.cl Villa, A. y Poblete, M. (directores) (2007) Aprendizaje basado en competencias. Una propuesta para la evaluación de las competencias genéricas; Ediciones Mensajero, Bilbao.

Recibido: 27/09/2010

Aceptado: 14/11/2010 


\section{Anexo A: Ítem Escala Frecuencia CACSR.}

\begin{tabular}{|c|c|}
\hline $\begin{array}{l}\text { Categorías de } \\
\text { Comportamiento }\end{array}$ & Ítem de Comportamiento \\
\hline \multirow{4}{*}{$\begin{array}{l}\text { Responsabilidad } \\
\text { académica }\end{array}$} & Asisto a clases \\
\hline & Cumplo responsablemente con tareas, trabajos y evaluaciones \\
\hline & Estudio las asignaturas de mi carrera \\
\hline & $\begin{array}{l}\text { Llego puntualmente a mis actividades académicas (reuniones de trabajo, } \\
\text { clases, prácticas profesionales, certámenes, etc.) }\end{array}$ \\
\hline \multirow[t]{4}{*}{$\begin{array}{l}\text { Actividades de } \\
\text { voluntariado }\end{array}$} & $\begin{array}{l}\text { Participo en actividades de voluntariado (construir mediaguas, visitar hogar } \\
\text { de niños o de ancianos, realizar trabajos estudiantiles de verano, etc.) }\end{array}$ \\
\hline & $\begin{array}{l}\text { Organizo campañas de ayuda solidaria (recolectar alimentos, vestimenta, } \\
\text { útiles escolares, etc.) }\end{array}$ \\
\hline & $\begin{array}{l}\text { Colaboro en la formación de otras personas (ayudo a mis compañeros a } \\
\text { estudiar, ayudo a niños con problemas de aprendizaje, enseño a adultos a } \\
\text { leer, etc.) }\end{array}$ \\
\hline & $\begin{array}{l}\text { Formo parte de alguna organización de ayuda social (Hogar de Cristo, } \\
\text { Fundación Las Rosas, Regazo). }\end{array}$ \\
\hline \multirow[t]{4}{*}{ Ayuda social } & Dono sangre \\
\hline & Dono dinero a campañas de ayuda social \\
\hline & $\begin{array}{l}\text { Compro productos que entregan un porcentaje de su utilidad a fundaciones } \\
\text { de ayuda social (tarjetas del Hogar de Cristo, souvenirs de la UNICEF, } \\
\text { calendarios scout, alimentos de Campeones para Chile, etc.) }\end{array}$ \\
\hline & $\begin{array}{l}\text { Entrego recursos materiales a campañas de ayuda social (vestimentas, } \\
\text { alimentos, materiales de construcción, etc.) }\end{array}$ \\
\hline \multirow{4}{*}{$\begin{array}{l}\text { Actividades } \\
\text { religiosas }\end{array}$} & Participo en algún grupo religioso (de cualquier religión) \\
\hline & Participo en las actividades propias de la religión que profeso \\
\hline & Invito a otras personas a participar en actividades de mi grupo religioso \\
\hline & Transmito los valores religiosos propios a otras personas \\
\hline \multirow[t]{4}{*}{$\begin{array}{l}\text { Convivencia } \\
\text { social }\end{array}$} & $\begin{array}{l}\text { Acepto a los demás independientemente de su sexo, raza, condición física, } \\
\text { orientación sexual, etc. }\end{array}$ \\
\hline & Escucho con atención a los demás cuando hablan \\
\hline & $\begin{array}{l}\text { Respeto normas sociales (respetar la fila, turnos, ceder el asiento a personas } \\
\text { mayores, etc.) }\end{array}$ \\
\hline & $\begin{array}{l}\text { Expreso mi opinión respecto a distintos temas, en diferentes contextos y con } \\
\text { diferentes personas }\end{array}$ \\
\hline \multirow{4}{*}{$\begin{array}{l}\text { Responsabilidad } \\
\text { cívico- } \\
\text { universitaria }\end{array}$} & $\begin{array}{l}\text { Pago la universidad (aranceles, crédito universitario, devolver el crédito, } \\
\text { matrícula y hacer uso honesto de las becas) }\end{array}$ \\
\hline & $\begin{array}{l}\text { Participo en movimientos estudiantiles tales como: federación de estudiantes o } \\
\text { centro de alumnos (asisto a asambleas, voto en elecciones universitarias, etc.) }\end{array}$ \\
\hline & Voto en elecciones presidenciales, parlamentarias, de alcaldes, etc. \\
\hline & $\begin{array}{l}\text { Exijo comprobante por servicios comerciales (boletas, boleto de micro, pasaje } \\
\text { de bus, etc.) }\end{array}$ \\
\hline
\end{tabular}


120 UNIVERSITARIOS Y RESPONSABILIDAD SOCIAL - Gracia Navarro, Paula Boero, Gladys Jiménez, Liliana Tapia, Reinier Hollander, Arturo Escobar, Margarita Baeza, y Álvaro Espina

\begin{tabular}{|c|c|}
\hline \multirow[t]{4}{*}{ Autocuidado } & $\begin{array}{l}\text { Cuido la salud, ya sea mediante una alimentación equilibrada, hacer deporte, } \\
\text { realizar controles médicos, evitar fumar, etc. }\end{array}$ \\
\hline & $\begin{array}{l}\text { Me procuro un espacio de recreación con los demás, ya sea compartiendo } \\
\text { con la familia, con los amigos(as), practicando deportes en equipo, etc. }\end{array}$ \\
\hline & Evito contagiarme de enfermedades de transmisión sexual \\
\hline & $\begin{array}{l}\text { Tengo espacios de recreación individuales (un hobby, ir al cine, escuchar } \\
\text { música, ver TV, etc.) }\end{array}$ \\
\hline \multirow[t]{4}{*}{$\begin{array}{l}\text { Desarrollo } \\
\text { cultural }\end{array}$} & $\begin{array}{l}\text { Me informo sobre el acontecer nacional e internacional (ver noticias, leer el } \\
\text { diario, etc.) }\end{array}$ \\
\hline & $\begin{array}{l}\text { Asisto a actividades para complementar la formación profesional (asistir a } \\
\text { charlas, seminarios o cursos, relacionados o no con la carrera) }\end{array}$ \\
\hline & Leo libros no relacionados con lo académico \\
\hline & $\begin{array}{l}\text { Participo en actividades culturales (ir a conciertos, ver obras de teatro, ir a } \\
\text { exposiciones de obras de pintura, escultura, etc.) }\end{array}$ \\
\hline \multirow[t]{4}{*}{$\begin{array}{l}\text { Ecología y } \\
\text { medioambiente }\end{array}$} & $\begin{array}{l}\text { Participo en campañas de cuidado del medioambiente (de recolección de } \\
\text { basura, limpieza de lugares públicos, etc.) }\end{array}$ \\
\hline & $\begin{array}{l}\text { Boto la basura en los basureros de la calle, de los parques o de lugares } \\
\text { públicos }\end{array}$ \\
\hline & $\begin{array}{l}\text { Utilizo racionalmente recursos naturales (agua, luz, gas, cuidar espacios } \\
\text { naturales, etc.) }\end{array}$ \\
\hline & $\begin{array}{l}\text { Evito usar productos que contaminen el ambiente (aerosoles, envases no } \\
\text { reciclables, tubos de escape en mal estado, etc.) }\end{array}$ \\
\hline \multirow{4}{*}{$\begin{array}{l}\text { Respeto por } \\
\text { los espacios } \\
\text { compartidos }\end{array}$} & Cuido espacios públicos (plazas, jardines, parques, monumentos, etc.) \\
\hline & Evito fumar en espacios públicos \\
\hline & Respeto espacios reservados para minusválidos, embarazadas y/o ancianos \\
\hline & $\begin{array}{l}\text { Cuido instalaciones públicas o universitarias (mobiliario, infraestructura, } \\
\text { libros, etc.) }\end{array}$ \\
\hline
\end{tabular}

Anexo B: Ejemplos de Ítem Escala Intencionalidad CACSR.

\begin{tabular}{|l|l|}
\hline Ítem de Comportamiento & Con la intención de \\
\hline Asisto a clases & Cumplir los requisitos de mi carrera y/o aprender \\
\cline { 2 - 2 } & Intercambiar conocimientos \\
\cline { 2 - 2 } & $\begin{array}{l}\text { Cumplir las expectativas de mis padres, profesores y/o personas } \\
\text { cercanas }\end{array}$ \\
\cline { 2 - 2 } & No tengo clara la intención \\
\hline $\begin{array}{l}\text { Participo en actividades } \\
\text { de voluntariado (construir } \\
\text { mediaguas, visitar hogar de } \\
\text { niños o de ancianos, realizar } \\
\text { trabajos estudiantiles de } \\
\text { verano, etc.) }\end{array}$ & Mejorar el bien común \\
\cline { 2 - 2 } Dono sangre & Obtener beneficios económicos y/o académicos la calidad de vida de las personas \\
\hline \multirow{2}{*}{$\begin{array}{l}\text { Nongo clara la intención } \\
\end{array}$} & Mantener las reservas de sangre en los hospitales del país \\
\cline { 2 - 2 } & Hacer un favor a un familiar, amigo y/o conocido que lo necesite \\
\cline { 2 - 2 } & Que exista sangre en caso que yo la necesite \\
\cline { 2 - 2 } & No tengo clara la intención \\
\hline
\end{tabular}




\begin{tabular}{|c|c|}
\hline \multirow{4}{*}{$\begin{array}{l}\text { Dono dinero a campañas de } \\
\text { ayuda social }\end{array}$} & Ayudar a los más necesitados \\
\hline & Sentirme bien \\
\hline & Disminuir la brecha entre clases sociales \\
\hline & No tengo clara la intención \\
\hline \multirow{4}{*}{$\begin{array}{l}\text { Participo en algún grupo } \\
\text { religioso (de cualquier } \\
\text { religión) }\end{array}$} & Compartir ideas, opiniones y sentimientos \\
\hline & Conocer gente \\
\hline & Ayudar a la formación espiritual de mis amigos (as) y familiares \\
\hline & No tengo clara la intención \\
\hline \multirow{4}{*}{$\begin{array}{l}\text { Acepto a los demás } \\
\text { independientemente de su } \\
\text { sexo, raza, condición física, } \\
\text { orientación sexual, etc. }\end{array}$} & $\begin{array}{l}\text { Que mis amigos (as), familiares y/o compañeros (as) no se sientan } \\
\text { marginados por alguna característica propia }\end{array}$ \\
\hline & Que exista mayor aceptación y tolerancia en la sociedad \\
\hline & Ser aceptado \\
\hline & No tengo clara la intención \\
\hline \multirow{4}{*}{$\begin{array}{l}\text { Pago la universidad } \\
\text { (aranceles, crédito } \\
\text { universitario, devolver el } \\
\text { crédito, matrícula y hacer uso } \\
\text { honesto de las becas) }\end{array}$} & Que mis familiares puedan estudiar \\
\hline & Estar tranquilo \\
\hline & Terminar con la deuda universitaria \\
\hline & No tengo clara la intención \\
\hline \multirow{4}{*}{$\begin{array}{l}\text { Evito contagiarme de } \\
\text { enfermedades de transmisión } \\
\text { sexual }\end{array}$} & $\begin{array}{l}\text { Disminuir la incidencia de enfermedades de transmisión sexual } \\
\text { en los jóvenes }\end{array}$ \\
\hline & Cuidar mi salud \\
\hline & Fomentar conductas similares en mis amigos (as) y/o familiares \\
\hline & No tengo clara la intención \\
\hline \multirow{4}{*}{$\begin{array}{l}\text { Me informo sobre el } \\
\text { acontecer nacional e } \\
\text { internacional (ver noticias, } \\
\text { leer el diario, etc.) }\end{array}$} & Aumentar mis conocimientos y/o tener una opinión informada \\
\hline & $\begin{array}{l}\text { Resolver dudas y explicar temas de actualidad a mis amigos (as), } \\
\text { familiares y/o compañeros (as) }\end{array}$ \\
\hline & Contribuir a resolver problemas de la sociedad \\
\hline & No tengo clara la intención \\
\hline \multirow{4}{*}{$\begin{array}{l}\text { Participo en campañas de } \\
\text { cuidado del medioambiente } \\
\text { (de recolección de basura, } \\
\text { limpieza de lugares públicos, } \\
\text { etc.) }\end{array}$} & $\begin{array}{l}\text { Que los niños crezcan en un entorno que les permita } \\
\text { desarrollarse }\end{array}$ \\
\hline & Disminuir la contaminación del planeta \\
\hline & Preservar y cuidar mis espacios \\
\hline & No tengo clara la intención \\
\hline \multirow{4}{*}{$\begin{array}{l}\text { Utilizo racionalmente } \\
\text { recursos naturales (agua, } \\
\text { luz, gas, cuidar espacios } \\
\text { naturales, etc.) }\end{array}$} & Yo pueda contar con ellos en el futuro \\
\hline & Preservar los recursos naturales del medioambiente \\
\hline & $\begin{array}{l}\text { Evitar el gasto innecesario de mis padres y/o personas que me } \\
\text { mantienen }\end{array}$ \\
\hline & No tengo clara la intención \\
\hline \multirow{4}{*}{$\begin{array}{l}\text { Respeto espacios reservados } \\
\text { para minusválidos, } \\
\text { embarazadas y/o ancianos }\end{array}$} & Sentirme bien y/o cumplir con mi obligación \\
\hline & Lograr una mejor convivencia social \\
\hline & $\begin{array}{l}\text { Que los minusválidos, embarazadas y/o ancianos de mi } \\
\text { comunidad se sientan respetados }\end{array}$ \\
\hline & No tengo clara la intención \\
\hline
\end{tabular}

